\title{
ON CERTAIN BASIC SERIES
}

JOHN A. DAUM

1. Introduction. The identity

(1) $\sum_{n=1}^{\infty} \frac{q^{n}}{\left(1-q^{n}\right)^{2}}\left\{\frac{1}{1-q}+\frac{1}{1-q^{2}}+\cdots+\frac{1}{1-q^{n}}\right\}=\sum_{n=1}^{\infty} \frac{n^{2} q^{n}}{1-q^{n}}$

was deduced from arithmetical considerations by E. T. Bell. About five years ago, W. N. Bailey ${ }^{1}$ proved the relation

(2) $\sum_{n=0}^{\infty} \frac{(1-q)\left(1-q^{2}\right) \cdots\left(1-q^{n}\right)}{(1-z)(1-q z) \cdots\left(1-q^{n} z\right)} \cdot \frac{z^{n+1}}{1-q^{n+1}}=\sum_{n=0}^{\infty} \frac{q^{n} z}{\left(1-q^{n} z\right)^{2}}$,

from which he obtained (1) by differentiating with respect to $z$ and then putting $z=q$. A short time later $\mathrm{Hall}^{2}$ gave an alternate proof of (2) by simply specializing the parameters in a relation between basic series. $^{3}$

Since Bell's identity (1) leads to interesting arithmetical results, it would be desirable to obtain as many more similar relations as possible. Hall's paper, as well as Bailey's, seems to indicate a connection with certain formulae in basic series. The identity which Hall employed to obtain Bailey's relation is the basic analogue of but one of many identities involving the series ${ }_{3} F_{2}$ which were proved originally by Thomae ${ }^{4}$ and later classified by Whipple. ${ }^{5}$

The object of this paper is to investigate the possibility of systematically obtaining new results similar to Bailey's by employing the complete set of basic analogues of Thomae's two-term transformations.

2. The basic analogues of Thomae's relations. We first establish the complete set of basic analogues of Thomae's relations, using a notation analogous to that used by Whipple.

Let $I=(a, b, c, d, e, f)$ be a matrix whose elements form an arbitrary permutation of the integers $0,1,2,3,4,5$ and let $r_{a}, r_{b}, r_{c}, r_{d}, r_{e}$, $r_{f}$ be six numbers such that their product is unity. Define parameters $A$ and $B$ by

${ }^{1}$ W. N. Bailey, An algebraic identity, Journal of the London Mathematical Society, vol. 11 (1936), p. 156.

${ }^{2}$ N. A. Hall, An algebraic identity, ibid., vol. 11 (1936), p. 276.

${ }^{3}$ W. N. Bailey, Generalized Hypergeometric Series, Cambridge Tract, no. 32, p. 65.

${ }^{4} \mathrm{~J}$. Thomae, Journal für die reine und angewandte Mathematik, vol. 87 (1879), p. 26.

${ }^{5}$ F. J. Whipple, Proceedings of the London Mathematical Society, vol. 23 (1925), p. 104. 


$$
A_{u v w}=r_{u} r_{v} r_{w} q^{1 / 2}, \quad|q|<1 ; \quad B_{u v}=r_{u} q / r_{v}
$$

and functions $\Phi p$ and $\Phi n$ by

$$
\begin{aligned}
& \Phi p(a ; b, c)=\frac{1}{\Omega\left(A_{d e f}\right) \Omega\left(B_{b a}\right) \Omega\left(B_{c a}\right)} \quad{ }_{s} \Phi_{2}\left[\begin{array}{cc}
A_{d b c}, & A_{e b c}, A_{f b c} ; A_{d e f} \\
B_{b a}, & B_{c a}
\end{array}\right],
\end{aligned}
$$

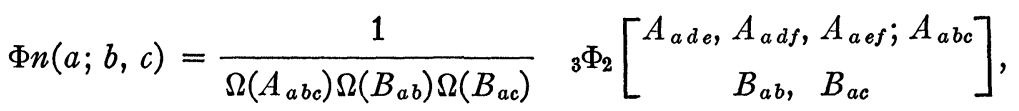

where ${ }^{6}$

$$
{ }_{3} \Phi_{2}\left[\begin{array}{c}
\alpha, \beta, \gamma ; z \\
\delta, \epsilon
\end{array}\right]=\frac{\Omega(\delta) \Omega(\epsilon)}{\Omega(\alpha) \Omega(\beta) \Omega(\gamma)} \sum_{n=0}^{\infty} \frac{\Omega\left(\alpha q^{n}\right) \Omega\left(\beta q^{n}\right) \Omega\left(\gamma q^{n}\right)}{\Omega\left(q^{n+1}\right) \Omega\left(\delta q^{n}\right) \Omega\left(\epsilon q^{n}\right)} z^{n}, \quad|z|<1,
$$

and $^{7}$

$$
\Omega(x)=\prod_{n=0}^{\infty} \frac{1-q^{n+1}}{1-x q^{n}} .
$$

Considering all possible matrices $I$, it is possible to form $60 \Phi p$ 's and $60 \Phi n$ 's.

There are 90 fundamental relations between certain pairs of the functions $\Phi p$. The proof of these relations, which can all be written in the form

$$
\Phi p(a ; b, c)=\Phi p(a ; d, e),
$$

is almost identical with that of the original case as given by Bailey, ${ }^{8}$ subject to certain analogous conditions. The only essential difference is in the replacement of the gamma-function throughout by the modified Heine omega-function.

The 90 identities (3) are all equivalent in the sense that any one of these identities may be obtained directly from any other by proper determination of the $r$ 's. The corresponding relations involving the functions $\Phi n$ are all equivalent to those involving the $\Phi p$ 's-one set being obtainable from the other by replacing the $r$ 's by their reciprocals.

As an example of the use of relations (3), consider the identity

$$
\Phi p(0 ; 4,5)=\Phi p(0 ; 2,3),
$$

where

${ }^{6}$ W. N. Bailey, Generalized Hypergeometric Series, Cambridge Tract, no. 32, p. 65.

7 N. A. Hall, loc. cit.

8 W. N. Bailey, Generalized Hypergeometric Series, Cambridge Tract, no. 32, p. 14. 
$A_{145}=\alpha, \quad A_{245}=\beta, \quad A_{345}=\gamma, \quad B_{40}=\delta, \quad B_{50}=\epsilon, \quad A_{123}=\delta \epsilon / \alpha \beta \gamma$.

We obtain the identity

$$
\begin{aligned}
{ }_{3} \Phi_{2}\left[\begin{array}{c}
\alpha, \beta, \gamma ; \delta \epsilon / \alpha \beta \gamma \\
\delta, \epsilon
\end{array}\right]= & \frac{\Omega(\delta) \Omega(\epsilon) \Omega(\delta \epsilon / \alpha \beta \gamma)}{\Omega(\alpha) \Omega(\delta \epsilon / \alpha \beta) \Omega(\delta \epsilon / \alpha \gamma)} \\
& \cdot{ }_{3} \Phi_{2}\left[\begin{array}{c}
\delta / \alpha, \epsilon / \alpha, \delta \epsilon / \alpha \beta \gamma ; \alpha \\
\delta \epsilon / \alpha \beta, \delta \epsilon / \alpha \gamma
\end{array}\right],
\end{aligned}
$$

which was used by Hall to obtain Bailey's identity (2).

For a fixed value of $a$ (3) represents 15 identities between certain of the functions $\Phi p(a)$. For $a=0$, these identities are

$$
\begin{array}{rlrl}
\Phi p(0 ; 4,5) & =\Phi p(0 ; 2,3) & \Phi p(0 ; 1,5) & =\Phi p(0 ; 2,3) \\
& =\Phi p(0 ; 1,3) & & =\Phi p(0 ; 2,4) \\
& =\Phi p(0 ; 1,2), & & =\Phi p(0 ; 3,4), \\
\Phi p(0 ; 3,5) & =\Phi p(0 ; 1,2) & \Phi p(0 ; 1,4) & =\Phi p(0 ; 2,3) \\
& =\Phi p(0 ; 1,4) & \Phi p(0 ; 2,4)=\Phi p(0 ; 1,3) \\
& =\Phi p(0 ; 2,4), & \Phi p(0 ; 3,4)=\Phi p(0 ; 1,2), \\
\Phi p(0 ; 2,5) & =\Phi p(0 ; 1,3) & & \\
& =\Phi p(0 ; 1,4) & \\
& =\Phi p(0 ; 3,4) . &
\end{array}
$$

From this, it is clear that we have a second set of identities of the form

$$
\Phi \cdot p(a ; b, c)=\Phi p(a ; b, d)
$$

subject to certain conditions obtained from those applied in proving (3). Relation (5) represents 180 equivalent identities. Typical of results obtained are

(6) ${ }_{3} \Phi_{2}\left[\begin{array}{c}\alpha, \beta, \gamma ; \delta \epsilon / \alpha \beta \gamma \\ \delta, \epsilon\end{array}\right]=\frac{\Omega(\delta) \Omega(\epsilon) \Omega(\delta \epsilon / \alpha \beta \gamma)}{\Omega(\delta \epsilon / \alpha \beta) \Omega(\epsilon) \Omega(\delta / \gamma)}{ }_{3} \Phi_{2}\left[\begin{array}{c}\epsilon / \beta, \epsilon / \alpha, \gamma ; \delta / \gamma \\ \delta \epsilon / \alpha \beta, \epsilon\end{array}\right]$

and

$$
\begin{aligned}
{ }_{3} \Phi_{2}\left[\begin{array}{c}
\delta \epsilon / \alpha \beta \gamma, \delta / \alpha, \epsilon / \alpha ; \alpha \\
\delta \epsilon / \alpha \gamma, \delta \epsilon / \alpha \beta
\end{array}\right]= & \frac{\Omega(\delta \epsilon / \alpha \gamma) \Omega(\delta \epsilon / \alpha \beta) \Omega(\alpha)}{\Omega(\delta \epsilon / \beta \gamma) \Omega(\delta \epsilon / \alpha \beta) \Omega(\beta)} \\
& { }_{3} \Phi_{2}\left[\begin{array}{c}
\delta \epsilon / \alpha \beta \gamma ; \delta / \beta, \epsilon / \beta ; \beta \\
\delta \epsilon / \beta \gamma, \delta \epsilon / \alpha \beta
\end{array}\right] .
\end{aligned}
$$


It is possible to obtain Bailey's identity from either (6) or ( 7 ).

3. Conclusion. This classification of the basic analogues of Thomae's two-term relations indicates, then, that out of the entire set of identities, only two are essentially distinct. Any further results can be obtained from this source only by specialization of the parameters in Hall's identity or in relation (6) or (7).

Analogous statements can be made concerning the corresponding relations between the generalized hypergeometric functions ${ }_{3} F_{2}$ as given by Whipple.

Agricultural and Mechanical College of Texas and

UNIVERSITY OF NEBRASKa

\section{A NOTE ON FINITE REGULAR RINGS}

\section{JOHN DYER-BENNET}

The purpose of this note is to give a classification of all finite regular rings. One result of the classification is the extension of a part of Wedderburn's classic theory of the structure of linear associative algebras to systems which are not algebras. I wish to thank Professor Garrett Birkhoff for several suggestions in the preparation of the note.

Definition. $A$ ring $R$ is said to be regular if for every element a of $R$ there exists an element $x$ in $R$ such that

$$
a k a=a .^{1}
$$

\section{Lemma 1. A regular ring has radical $(0){ }^{2}$}

Proof. Since the radical of a ring is a nilpotent ideal, any element $a$ in it has the property that a product containing $a$ as a factor a sufficient number of times is zero. Since by definition there exists an $x$ such that $a x a x \cdots a x a=a$ no matter how many terms there are in the product, zero must be the only member of the radical.

${ }^{1}$ Cf. von Neumann, Lectures on Continuous Geometries, Part 2, Princeton, 1937, pp. 7-21. Also On regular rings, Proceedings of the National Academy of Sciences, vol. 22 (1936), pp. 707-713. von Neumann postulates the existence of a unit, but in our case this follows from the other assumptions.

${ }^{2}$ Cf. von Neumann, loc. cit. 